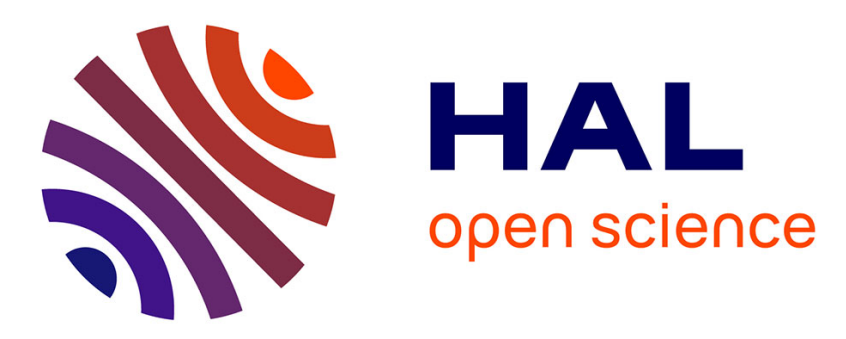

\title{
Shock wave generation triggered by a weak background in optical fibers
}

G. Xu, Arnaud Mussot, Alexandre Kudlinski, Stefano Trillo, F. Copie, Matteo

Conforti

\section{- To cite this version:}

G. Xu, Arnaud Mussot, Alexandre Kudlinski, Stefano Trillo, F. Copie, et al.. Shock wave generation triggered by a weak background in optical fibers. Optics Letters, 2016, 41 (11), pp.2656-2659. 10.1364/OL.41.002656 . hal-02386570

\section{HAL Id: hal-02386570 https://hal.science/hal-02386570}

Submitted on 29 Nov 2019

HAL is a multi-disciplinary open access archive for the deposit and dissemination of scientific research documents, whether they are published or not. The documents may come from teaching and research institutions in France or abroad, or from public or private research centers.
L'archive ouverte pluridisciplinaire HAL, est destinée au dépôt et à la diffusion de documents scientifiques de niveau recherche, publiés ou non, émanant des établissements d'enseignement et de recherche français ou étrangers, des laboratoires publics ou privés. 
archives-ouvertes

\title{
Shock wave generation triggered by a weak background in optical fibers
}

\author{
G. Xu, A. Mussot, A. Kudlinski, S. Trillo, F. Copie, M. Conforti
}

\section{To cite this version:}

G. Xu, A. Mussot, A. Kudlinski, S. Trillo, F. Copie, et al.. Shock wave generation triggered by a weak background in optical fibers. Optics Letters, Optical Society of America, 2016, 41 (11), pp.2656. 10.1364/OL.41.002656 . hal-02386570

\section{HAL Id: hal-02386570 \\ https://hal.archives-ouvertes.fr/hal-02386570}

Submitted on 29 Nov 2019

HAL is a multi-disciplinary open access archive for the deposit and dissemination of scientific research documents, whether they are published or not. The documents may come from teaching and research institutions in France or abroad, or from public or private research centers.
L'archive ouverte pluridisciplinaire HAL, est destinée au dépôt et à la diffusion de documents scientifiques de niveau recherche, publiés ou non, émanant des établissements d'enseignement et de recherche français ou étrangers, des laboratoires publics ou privés. 


\title{
Shock wave generation triggered by a weak background in optical fibers
}

\author{
G. Xu, ${ }^{1}$ A. Mussot, ${ }^{1, *}$ A. Kudlinski, ${ }^{1}$ S. Trillo, ${ }^{2}$ F. Copie, ${ }^{1}$ M. Conforti ${ }^{1}$ \\ ${ }^{1}$ Univ. Lille, CNRS, UMR 8523 - PhLAM - Physique des Lasers Atomes et Molécules, F-59000 Lille, France \\ ${ }^{2}$ Department of Engineering, University of Ferrara, Via Saragat, 44122 Ferrara, Italy \\ *arnaud.mussot@univ-lille1.fr
}

Compiled April 12, 2016

We experimentally report the observation of dispersive shock waves from a short pulse superimposed onto a small continuous wave background in optical fibers. We show that the background allows to strongly enhance the extension and contrast of the oscillatory wave train inherent to the dispersive shock. More than 7 periods of oscillations with a high contrast are observed experimentally and confirmed with numerical simulations. The dynamics of the process is simply explained from spectro-temporal representations.

OCIS codes: (190.4370) Nonlinear optics, fibers; (190.5530) Pulse propagation and temporal solitons.

http://dx.doi.org/10.1364/ao.XX.XXXXXX

Shock waves have been thoroughly investigated during the last century in many different branches of physics [1]. In dissipative systems, viscosity regularises the shock, determining its finite width. In conservative systems, a different regulariza-tion occurs which entails the formation, owing to dispersion, of rapidly oscillating non-stationary structures, called undular bores (UBs) or dispersive shock waves (DSWs). Originally studied in plasmas [3] and water waves [4,5], DSWs are now the subject of intense theoretical and experimental studies in different areas of physics (for a review see [2]).

In optics DSWs have been the focus of recent investigations both in the nonlinear spatial beam propagation in defocusing media [6-9] and in time domain exploiting different fiber op-tics configurations [10-17]. In optical fibers, DSWs result from the combined effects of self-phase modulation (SPM) and nor-mal group velocity dispersion (GVD) leading to the formation of sharp pulse edges and then to characteristic oscillations on the wings of the pulse [18, 19]. However, early observations in fibers made in the late 80 's, which investigated the wave breaking by means of spectro-temporal techniques (streak-camera spectrogram) [20] and autocorrelations [21], reported only a few intensity oscillations with a very low contrast. This is due to the fact that the measurements were performed only for standard pulses with zero pedestal. Conversely, a non-zero background is expected to strongly enhance the DSW both in terms of its temporal extension and the contrast of the fast oscillations. This conclusion mostly follows from (Whitham) modulation theory [2], which allows to analytically describe the asymptotic decay of initial intensity jump ruled by the defocusing nonlinear Schrödinger equation (NLSE), the so-called Riemman problem $[22,24]$. The outcome is that an even small background of the input pulse results into an increase of the amplitude and contrast of the expanding intensity modulation appearing after the breaking of the wave. An indirect indication of that appears in the spatial experiments involving photorefractive screening nonlinearities, intrinsically charactecterised by a background illumination [6]. However, systematic experimental studies of this problem have never been reported. In this letter, we experimentally observe the formation of temporal DSWs from an optical pulse resting on a weak background at the fiber input. Our set-up allows to assess the impact of the background, showing that the contrast of the oscillations in the pulse tails associated with the wave breaking is strongly enhanced and eventually approaches unity.

We first start by presenting numerical investigations based on the one dimensional NLSE:

$$
\partial_{z} A=-\frac{i \beta_{2}}{2} \partial_{t t} A+i \gamma|A|^{2} A-\frac{\alpha}{2} A,
$$

where $A$ is the envelope amplitude, $z$ is the distance, $t$ is the retarded time, $\beta_{2}$ the dispersion, and $\gamma$ the nonlinear coefficient. In order to highlight the role played by a small background on the formation of high contrast oscillations due to wave breaking, we perform numerical simulations from Eq. (1). In Fig. 1(a) and (b) we contrast the longitudinal intensity evolution for an input Gaussian pulse launched with and without background in an optical fiber. Initial conditions are shown in Fig. 1(c) and (d) as dashed black lines. The background level is fixed to $10 \%$ of the peak power of the input pulse. Up to about $800 \mathrm{~m}$, no significant difference appears between these two configurations. The Gaussian pulse broadens to a rectangular-like pulse with sharp edges through the combined actions of dominant SPM and weak GVD [see solid blue lines in Fig. 1(c) and (d)]. This is the typical wave breaking process observed in normally dispersive optical fibers. By further propagating along the fiber, the impact of the background becomes visible. Rapid oscillations with a high contrast appear when the pulse seats on a background [Fig. 1(b)] while, in the absence of the latter, the pulse exhibits a non-oscillating monotone decay [Fig. 1(a)]. This is 
even clearer by contrasting Fig. 1(c) and (d), where the fiber output intensities are displayed. Oscillations with an average period 4.8 ps are generated with a high contrast (the period of the oscillation scales as $t_{0} \sqrt{L_{n l} / L_{d}}=\sqrt{\beta_{2} /(\gamma P)}$, where $t_{0}$ is the input pulse duration and $L_{n l}=1 / \gamma P$ and $L_{d}=t_{0}^{2} / \beta_{2}$ are the nonlinear and dispersion length, respectively, constrained to be in the regime $L_{n l} \ll L_{d}$ for the shock to take place). The losses have a very little impact, visible on the background in Fig. 1 (d), but do not appreciably affect the shock dynamics. We have also checked that, in the regime considered here, other effects such as steepening, Raman scattering and higher order dispersion can be safely neglected.
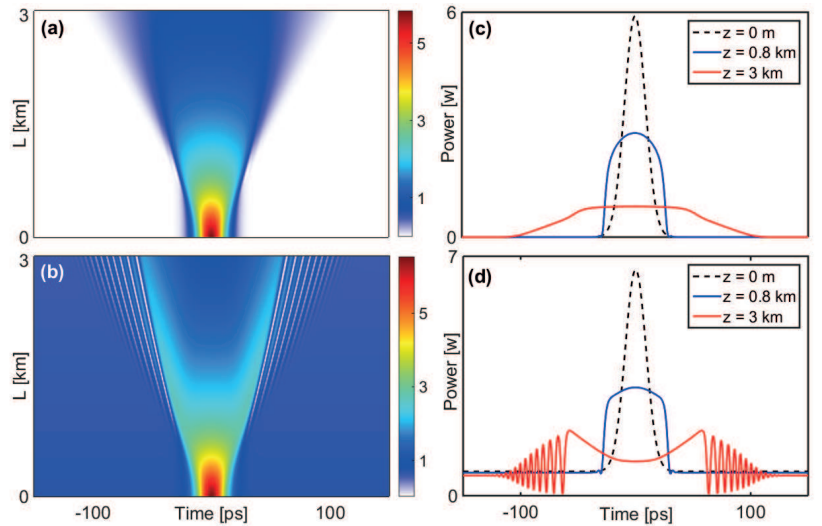

Fig. 1. (a-b) Level color-plot of power evolution (a) without and (b) with background, respectively, from numerical solution of Eq. (1). (c,d) intensity profiles for both cases at the output (red solid lines) and breaking distance $z=0.8 \mathrm{~km}$ (blue solid lines). The Gaussian input pulse (black dashed line) $A(t, 0)=\sqrt{P} \exp \left(-t^{2} / t_{0}^{2}\right)$ with $P=5.9 \mathrm{~W}$ peak power and full width at half maximum duration $t_{F W H M}=\sqrt{2 \ln (2)} t_{0}=$ $21.5 \mathrm{ps}$, is superimposed on a background with power $0.6 \mathrm{~W}$. Fiber parameters: $\beta_{2}=2.5 \times 10^{-26} \mathrm{~s}^{2} / \mathrm{m}, \gamma=3(\mathrm{~W} \cdot \mathrm{km})^{-1}$, $\alpha=0.26 \mathrm{~dB} / \mathrm{km}$.

The illustrated behaviour is consistent with theoretical predictions based on the assumption to start with a jump in intensity, i.e. the Riemann problem. First, it is well known that the shock formation dynamics is well described by the shallow water equations which is the leading order reduction for the NLSE [15, 22-24]. The jump problem for such model produces a shock only when a background (non-zero intensity over the lower state of the jump) is present, otherwise the solution is smooth [23]. Second, the onset of oscillations that regularises the gradient catastrophe can be described in the context of modulation theory, pioneered in $[22,24]$ in the context of dispersive hydrodynamics. Such calculations show that the amplitude of the oscillations reduces to zero in the limit of zero background, similarly to what is illustrated in Fig. 1(a,c). However, this asymptotic theory does not strictly apply to smooth pulse inputs and requires cumbersome calculations. Therefore we prefer to stick here to a more immediate interpretation, which allows for a more accessible physical insight in terms of chirp effects induced by GVD and SPM in fibers $[19,21]$.

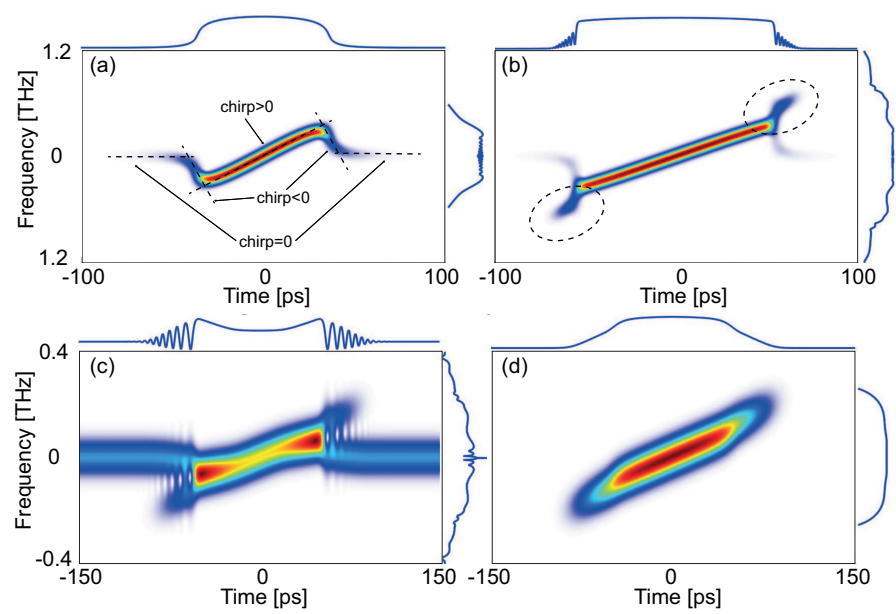

Fig. 2. Spectrograms with different input pulses: (a,b) sechshaped pulse, $t_{F W H M}=32$ ps duration, $P=80 \mathrm{~W}$ peak power, at $z=368 \mathrm{~m}$ and $710 \mathrm{~m}$ respectively; (c,d) Gaussian pulse with $t_{F W H M}=21.5$ ps (same as in Fig. 1): (c) with background and (d) without background at $z=3 \mathrm{~km}$. Fiber parameters as in Fig. 1 and in the experiment. The gate duration used to build the spectrograms is $6 \mathrm{ps}$. Movies are available online to see the evolution as a function of the fiber length.

The first stage of the propagation is turned out to be dominated by nonlinear effects. The SPM process leads to a new repartition of the energy in the spectrum with a positive chirp in the central part of the pulse, negative chirp in the leading and trailing edges and zero chirp in the vicinity of the pulse background. Note that these components have exactly the same frequency as the central frequency of the input signal. This is illustrated in Fig. 2 (a), where for example, the spectrogram of a powerful hyperbolic secant pulse (peak power $80 \mathrm{~W}$ and 32 ps duration at FWHM) without background is shown. By further propagating within the fiber, dispersion effects become significant and induce a positive chirp over all the pulse. As a consequence, the edges of the pulse become extremely sharp leading to the generation of very high frequencies, higher that those induced by the initial SPM effect (highlighted by circles in dashed lines in Fig. 2 (b)). They are located in the same temporal region than the spectral components with zero chirp and the same frequency than the input pulse. They can thus interfere leading to the formation of a standard interference pattern, whose frequency is equal to their frequency difference, and the contrast to their amplitude ratio. As can be see in Fig. 2 (b), these parameters evolve along the interference pattern. The period decreases with the time shift because a positive chirp is associated to the generation of high frequencies on the leading and trailing edges of the pulse. Concerning the contrast, it decreases and tends toward zero because from about $70 \mathrm{ps}$, the energy of the spectral components with zero chirp almost vanishes.

In this example, only a few periods of oscillations can be seen with a relatively weak contrast and amplitude despite we used a sech-shape as initial condition to enhance them. Indeed, compared to, e.g. Gaussian pulses with the same FWHM duration, sech-shape pulses have smoother trailing edges which, through the action of SPM, lead to an increase of the amount of energy of spectro-temporal components with zero chirp involved in the formation of the interference pattern. It is then not trivial to generate high contrast oscillations associated with 


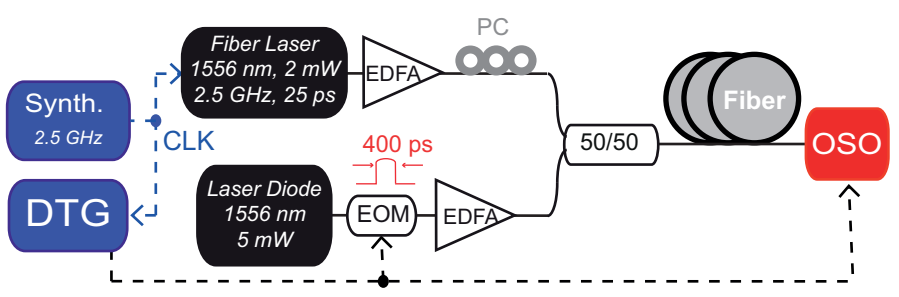

Fig. 3. Experimental setup. EDFA: erbium doped fiber amplifier, PC: polarization controller, OSO: optical sampling oscilloscope, DTG: data timing generator.

the shock wave formation by using a single pulse, as it is illustrated by the literature on this topic (see Refs $[15,18,21]$ and references therein for example). Adding a weak continuous wave background with same frequency than the input pulse, would "artificially" increase the amount of energy of these components involved in the interference pattern formation. Consequently, the visibility of the interference pattern would be strongly enhanced. This is illustrated in Figs. 2(c) and (d) where output spectrograms are displayed for an input Gaussian pulse with and without background, respectively. Without background, characteristic shoulders of a shock formation appear on the edges of the pulse but with no oscillations while with the background high contrast oscillations are formed.

We performed a series of experiments to illustrate the key role played by the initial continuous background in the wave breaking process. The parameters are the same as those used in the simulations and listed in the caption of Fig. 1. A sketch of the experimental setup is shown in Fig. 3. We used an actively mode-locked laser delivering Gaussian pulses of 21.5 ps FWHM duration and $2.5 \mathrm{GHz}$ repetition rate. The central wavelength of the laser is fixed to $1556 \mathrm{~nm}$. These pulses are amplified passing through an EDFA and are then combined with square pulses of 400 ps duration (more than ten times the initial pulse duration) located at the same wavelength, acting as the background. They are generated from an intensity modulator and amplified by a second EDFA. In this way stimulated Brillouin scattering effect is avoided and relatively high background powers are available. A careful synchronization be tween the laser and the square pulses is assured by a DTG that drives the intensity modulator. Both equipments are driven with the same clock that originates from an-ultra-low noise frequency synthetizer. We estimated that the timing jitter is lower than 1 ps. The polarization state of the pulses is controlled by using a polarization controller and the pulse resting on the background is launched along a normally dispersive optical fiber. Output results are analysed in the temporal domain by using an optical sampling oscilloscope with a 1.3 ps resolution (Eyechecker, Alnair).

We recorded the evolution of the intensity profiles as a function of the input pulse peak power at the output of the fiber, with and without background. In these experiments, the background level is equal to $10 \%$ of the maximum value of the input pulse peak power. Experimental results are shown in Figs. 4(c) and (d) (without and with background, respectively), and the input power varies from $0.6 \mathrm{~W}$ to $5.9 \mathrm{~W}$. Without the background, a plateau is generated on the top of the pulse even for lowest power values (Fig. 4(c)). Increasing the pump power leads to a temporal broadening of the pulse with the appear-
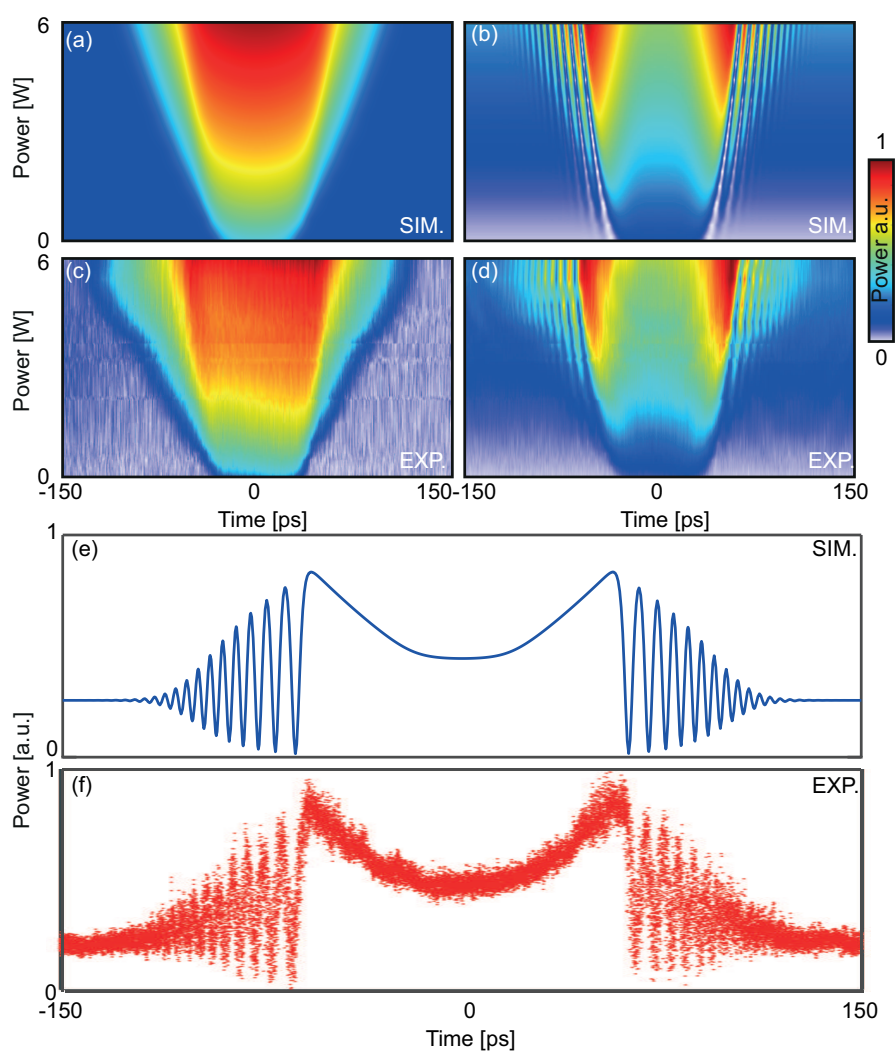

Fig. 4. Map of the output temporal profiles versus input pulse peak power: $(b, d)$ with background; $(a, c)$ without background. The background level is fixed to $10 \%$ of the input pulse peak power. $(\mathrm{e}, \mathrm{f})$ intensity profile corresponding the maximum input pulse peak power $(P=5.9 \mathrm{~W})$ from simulation and experiment, respectively. $(\mathrm{a}, \mathrm{b}, \mathrm{e})$ simulations; $(\mathrm{c}, \mathrm{d}, \mathrm{f})$ experiments.

ance of shoulders around the pulse without any intensity oscillation indicating that we are just at the onset of the wave breaking phenomenon [21]. In the presence of a weak background (Fig. 4(d)), the dynamics is similar for low power values. However, for higher power values, from about $4 \mathrm{~W}$, once the pulse experiences a large broadening through the action of dispersion, fast oscillations appear on the pulse tails [see Fig. 4(d)]. In order to get a clearer picture of these oscillations, the intensity profile at the fiber output obtained with the maximum input pulse peak power $(5.9 \mathrm{~W})$ is displayed in Fig. 4 (f). More than seven periods of oscillation with a high contrast are induced by the background. This clearly confirms that adding a weak background at the fiber input allows to increase the visibility of the underlying interference process and thus the contrast of the oscillations nearby the tails of the pulse. All these experimental results are in excellent agreement with corresponding numerical simulations (compare Fig. 4(e) to (f), and Fig. 4 (b) to (d)). In Refs. [22, 24], it is theoretically demonstrated that increasing the background level leads to an increase of the global contrast of the oscillations. As discussed above and illustrated in Fig. 2 with spectrograms, this can be explained as a basic interference process. In order to further validate it experimentally, we fixed the input pulse peak power to $5.9 \mathrm{~W}$ and we varied the background level from 0 to $10 \%$. A summary of the results is shown in Fig 5(a), where we plot the contrast as a function of 

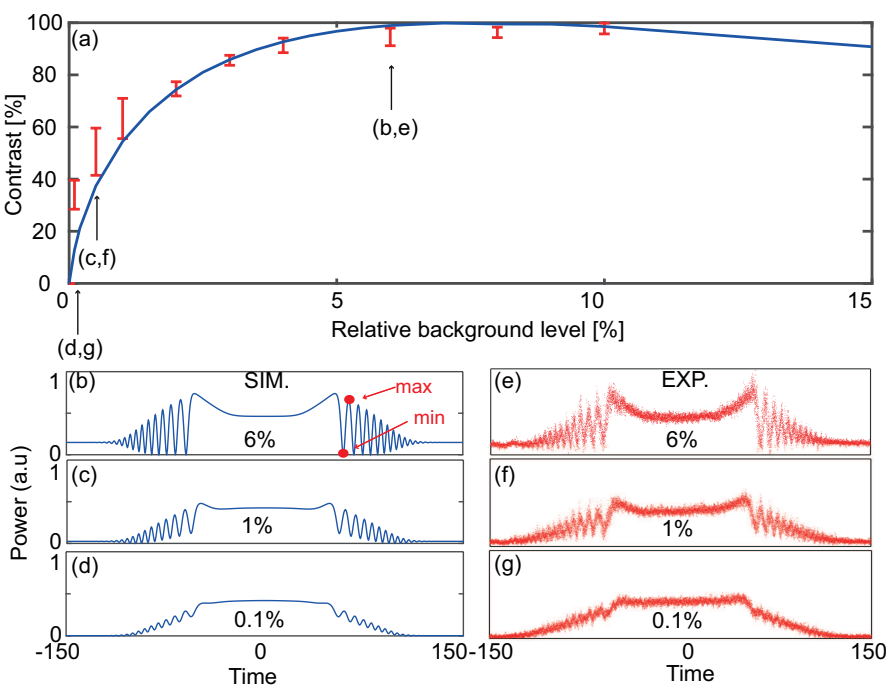

Fig. 5. (a) Oscillation contrast versus relative background level. (b) to (g): examples of different intensity profiles for $0.1,1$ and $6 \%$ relative background level: (b) to (d) numerical simulations; (e) to (g) experimental results.

the relative background level . The blue curve represents the results obtained from numerical simulations and the dots with error bars stand for the experimental results. We use the standard definition of the contrast $C$, as in standard interferometers:

$$
C=\frac{P_{\max }-P_{\min }}{P_{\max }+P_{\min }}
$$

with $P_{\max \text {, min }}$ the maximum (minimum) power of the first oscillation period (see Fig. 5 (b)). As can be seen in Figs. 5 (b)-(g), the contrast evolves along the tails of the pulse. In order to simply illustrate its evolution, we arbitrarily defined it for the first oscillation period, i.e. the leading edge of the DSW [see Fig. 5(b)]. Compared to other periods, this choice has the advantage of reducing the uncertainties in the measurements extracted from experimental traces since it usually exhibits the highest contrast, though the evolution would be very similar by taking another reference. As can be seen in Fig. 5 (a), it increases from 0 to nearly $100 \%$ by increasing the background level from 0 to $10 \%$. A few examples corresponding to $0.1,1,6 \%$ are illustrated in Figs. 5 (b-g). In particular, Figs. $5(\mathrm{~b}-\mathrm{d})$ show numerical simulations corresponding to the experimentally recorded profiles shown in Figs. 5(e-f-g). In these figures, an excellent agreement between simulations and experiments is obtained for the overall pulse shape.

We point out that a $100 \%$ contrast is reached over the flattop maximum of the solid curve in Fig. 5(a), in a range of background values nearly equal to $6-10 \%$, where the leading edge of the DSW turns out to have a vanishing intensity $\left(P_{\min }=0\right)$. Above such value the contrast slowly decreases as the curve in Fig. 5(a) clearly indicates. This is due to the fact that, at sufficiently large backgrounds, the leading edge of the DSW detaches again from zero and very slowly increases, inducing a smooth reduction of the contrast. Such behaviour is in qualitative agreement with the analytical results from the modulation theory for the Riemann problem (evolution of a jump) [24]. In the experiment, however, we only reach contrasts around the maximum, due to limitation in power of the background.
To summarize, we experimentally observed the formation of a DSW from a short pulse superimposed onto a weak background in an optical fiber. Adding a weak background to the input pulse results in a substantial enhancement of the contrast of fast oscillations inherent to the dispersive wave breaking, which can be understood in terms of interference process using spectrograms. Our experimental results have been confirmed with numerical simulations. This observation allows for a deeper understanding of applications based on wave breaking $[12,17]$, and to figure out new experiments aimed at investigating new hydrodynamical regimes in classical and quantum systems ruled by the NLSE.

\section{FUNDING INFORMATION}

The present research was partially supported by IRCICA (USR 3380 CNRS), by the Agence Nationale de la Recherche in the framework of the Labex CEMPI (ANR-11-LABX-0007-01), Equipex FLUX (ANR-11-EQPX-0017), by the projects NoAWE (ANR-14-ACHN-0014), TOPWAVE (ANR-13-JS04-0004), and FOPAFE (ANR-12-JS09-0005). S.T. acknowledges also the grant PRIN 2012BFNWZ2. We are grateful to J. Fatome and A. Picozzi for the fruitful discussions. We also acknowledge R. Kassi and L. Bigot for technical support about the electronic devices.

\section{REFERENCES}

1. G.B.Whitham, Linear and Nonlinear Waves (Wiley, New York, 1974)

2. G. A. El and M. A. Hoefer, "Dispersive shock waves and modulation theory", arXiV:1602.06163v1 [nlin.PS]

3. R.Z. Sagdeev, Sov. Phys. Tech. Phys. 6, 867 (1962).

4. D. H. Peregrine, J. Fluid Mech. 25, 321 (1966).

5. J. L. Hammack and H. Segur, J. Fluid Mech. 65, 289 (1974).

6. W. Wan, S. Jia, and J. W. Fleischer, Nature Phys. 3, 46 (2006).

7. N. Ghofraniha, C. Conti, G. Ruocco, and S. Trillo, Phys. Rev. Lett. 99, 043903 (2007).

8. C. Conti, A. Fratalocchi, M. Peccianti, G. Ruocco, and S. Trillo, Phys. Rev. Lett. 102, 083902 (2009).

9. G. Xu, D. Vock, D. Faccio, J. Garnier, T. Roger, S. Trillo, and A. Picozzi, Nature Commm. 6, 8131 (2015).

10. C. Conti, S. Stark, P. St. J. Russell, and F. Biancalana, Phys. Rev. A 82, 013838 (2010).

11. Y. Liu, H. Tu, and S. A. Boppart, Opt. Lett. 37, 2172 (2012).

12. B. Varlot, S. Wabnitz, J. Fatome, G. Millot, and C. Finot, Opt. Lett. 38, 3899 (2013).

13. M. Conforti, S. Trillo, Opt. Lett. 38, 3815 (2013).

14. J. Garnier, G. Xu, S. Trillo, and A. Picozzi, Phys. Rev. Lett. 111, 113902 (2013).

15. J. Fatome, C. Finot, G. Millot, A. Armaroli and S. Trillo, Phys. Rev. X 4, 021022 (2014).

16. M. Conforti, A. Mussot, A. Kudlinski, and S. Trillo, Sci. Rep. 5, 9433 (2015).

17. G. Millot, S. Pitois, M. Yan, T. Hovhannisyan, A. Bendahmane, T.W. Hänsch, and N. Picqué, Nat. Photon. 10, 27 (2016).

18. W. J. Tomlinson, R. H. Stolen and A. M. Johnson, Opt. Lett. 10, 457 (1985).

19. D. Anderson, M. Desaix, M. Lisak, and M. L. Quiroiga-Teixeiro, J. Opt. Soc. Am. B 9, 1358 (1992).

20. J.-P. Hamaide and P. Emplit, Electron. Lett. 24, 819 (1988).

21. J. Rothenberg and D. Grischkowsky, Phys. Rev. Lett. 62, 531 (1989).

22. A. Gurevich and A. Krylov, JETP 5, 65 (1987).

23. Y. Kodama and S. Wabnitz, Opt. Lett. 20, 2291 (1995).

24. G. El, V. Geogjaev, A. Gurevich, and A. Krylov, Physica D 87, 186 (1995). 


\section{REFERENCES}

1. G.B.Whitham, Linear and Nonlinear Waves (Wiley, New York,1974)

2. G. A. El and M. A. Hoefer, "Dispersive shock waves and modulation theory", arXiV:1602.06163v1 [nlin.PS]

3. R.Z. Sagdeev, "The Fine Structure of a Shock Wave Front Propagated across a Magnetic Field in a Rarefied Plasma", Sov. Phys. Tech. Phys. 6, 867 (1962).

4. D. H. Peregrine, "Calculations of the development of an undular bore", J. Fluid Mech. 25, 321 (1966).

5. J. L. Hammack and H. Segur, "The Korteweg-de Vries equation and water waves. Part 2. Comparison with experiments ", J. Fluid Mech. 65, 289 (1974).

6. W. Wan, S. Jia, and J. W. Fleischer, "Dispersive superfluid-like shock waves in nonlinear optics", Nature Phys. 3, 46 (2006).

7. N. Ghofraniha, C. Conti, G. Ruocco, and S. Trillo, "Shocks in nonlocal media", Phys. Rev. Lett. 99, 043903 (2007).

8. C. Conti, A. Fratalocchi, M. Peccianti, G. Ruocco, and S. Trillo, "Observation of a gradient catastrophe generating solitons", Phys. Rev. Lett. 102, 083902 (2009).

9. G. Xu, D. Vock, D. Faccio, J. Garnier, T. Roger, S. Trillo, and A. Picozzi, "From coherent shocklets to giant collective incoherent shock waves in nonlocal turbulent flows", Nature Commm. 6, 8131 (2015).

10. C. Conti, S. Stark, P. St. J. Russell, and F. Biancalana, "Multiple hydrodynamical shocks induced by the Raman effect in photonic crystal fibres," Phys. Rev. A 82, 013838 (2010).

11. Y. Liu, H. Tu, and S. A. Boppart, "Wave-breaking-extended fiber supercontinuum generation for high compression ratio transform-limited pulse compression", Opt. Lett. 37, 2172 (2012).

12. B. Varlot, S. Wabnitz, J. Fatome, G. Millot, and C. Finot, "Experimental generation of optical flaticon pulses", Opt. Lett. 38, 3899 (2013).

13. M. Conforti, S. Trillo, "Dispersive wave emission from wave breaking", Opt. Lett. 38, 3815 (2013)

14. J. Garnier, G. Xu, S. Trillo, and A. Picozzi, "Incoherent dispersive shocks in the spectral evolution of random waves", Phys. Rev. Lett. 111, 113902 (2013).

15. J. Fatome, C. Finot, G. Millot, A. Armaroli and S. Trillo, "Observation of optical undular bores in multiple four-wave mixing", Phys. Rev. X 4, 021022 (2014).

16. M. Conforti, A. Mussot, A. Kudlinski, and S. Trillo, "Parametric excitation of multiple resonant radiations from localized wavepackets", Sci. Rep. 5, 9433 (2015).

17. G. Millot, S. Pitois, M. Yan, T. Hovhannisyan, A. Bendahmane, T.W. Hänsch, and N. Picqué, Nat. Photon. 10, 27 (2016).

18. W. J. Tomlinson, R. H. Stolen and A. M. Johnson, "Optical wave breaking of pulses in nonlinear optical fibers", Opt. Lett. 10, 457 (1985).

19. D. Anderson, M. Desaix, M. Lisak, and M. L. Quiroiga-Teixeiro, "Wave breaking in nonlinear-optical fibers", J. Opt. Soc. Am. B 9, 1358 (1992).

20. J.-P. Hamaide and P. Emplit, "Direct observation of optical wave breaking of picosecond pulses in nonlinear single-mode optical fibres", Electron. Lett. 24, 819 (1988).

21. J. Rothenberg and D. Grischkowsky, "Observation of the Formation of an Optical Intensity Shock and Wave Breaking in the Nonlinear Propagation of Pulses in Optical Fibers", Phys. Rev. Lett. 62, 531 (1989).

22. A. Gurevich and A. Krylov, "Dissipationless shock waves in media with positive dispersion", JETP 5, 65 (1987).

23. Y. Kodama and S. Wabnitz, "Analytical theory of guiding-center nonreturn-to-zero and return-to-zero signal transmission in normally dispersive nonlinear optical fibers", Opt. Lett. 20, 2291 (1995).

24. G. El, V. Geogjaev, A. Gurevich, and A. Krylov, "Decay of an initial discontinuity in the defocusing NLS hydrodynamics", Physica D 87, 186 (1995). 